\title{
Challenges to the Israeli healthcare system: attracting medical students to primary care and to the periphery
}

\author{
Charles Weissman ${ }^{1 *}$, Rachel Yaffa Zisk-Rony ${ }^{2}$, Alexander Avidan ${ }^{1}$, Uriel Elchalal ${ }^{3}$ and Howard Tandeter $^{4}$
}

\begin{abstract}
Background: The greatest challenges facing healthcare systems include ensuring a sufficient supply of primary care physicians and physicians willing to work in rural or peripheral areas. Especially challenging is enticing young physicians to practice primary care in rural/peripheral areas. Identifying medical students interested in primary care and in residencies in Israel's periphery should aid the healthcare leadership. It may be particularly important to do so during the clinical years, as this is the stage at which many future physicians begin to crystallize their specialty and location preferences.

Methods: Questionnaires, distributed to 6 consecutive 5th-year classes of the Hebrew University - Hadassah School of Medicine, from 2010 to 2016, elicited information on criteria for choosing a career specialty, criteria for choosing a residency program and whether one-time monetary grants authorized in the 2011 physicians' union contract would attract students to residencies in the periphery.

Results: Completed questionnaires were returned by 511 of 740 (69\%) students. Ninety-eight (19\%) were interested in a primary care residency, 184 (36\%) were unsure and 229 (45\%) were not interested. Students interested in primary care were significantly less interested in specialties that perform procedures/surgeries and in joining a medical school faculty, while being more inclined towards specialties dealing with social problems, controllable lifestyles and working limited hours. The percentage of students interested in primary care was stable during the study period.

Forty-eight of the students indicated interest in residencies in the country's periphery, and $42 \%$ of them were also interested in primary care residencies. Overall, only $3.7 \%$ of students were interested in both a primary care residency and a residency in the periphery.

Thirty percent of the students indicated that the monetary incentives tempted them to consider a residency in the periphery. Fifty-three percent of these students reported that they did not yet know the geographic area where they wished to do their residency, as compared to only $22 \%$ among those not interested in incentives.

(Continued on next page)
\end{abstract}

\footnotetext{
* Correspondence: charles@hadassah.org.il

'Department of Anesthesiology and Critical Care Medicine,

Hadassah-Hebrew University Medical Center, Hebrew University - Hadassah

School of Medicine, Kiryat Hadassah, POB 12000, 91120 Jerusalem, Israel

Full list of author information is available at the end of the article
}

(c) The Author(s). 2018 Open Access This article is distributed under the terms of the Creative Commons Attribution 4.0 International License (http://creativecommons.org/licenses/by/4.0/), which permits unrestricted use, distribution, and reproduction in any medium, provided you give appropriate credit to the original author(s) and the source, provide a link to the Creative Commons license, and indicate if changes were made. The Creative Commons Public Domain Dedication waiver (http://creativecommons.org/publicdomain/zero/1.0/) applies to the data made available in this article, unless otherwise stated. 
(Continued from previous page)

Conclusions: This study provides the healthcare leadership with information on the characteristics of the students at a centrally-located medical school who tend to be more interested in primary care and in working in the periphery. Specifically, the study found that students interested in primary care desire a positive life/work balance, something that Israeli non-hospital primary care practice provides. Students considering residencies in the periphery were similarly inclined. Moreover, about a third of students had positive thoughts about monetary incentives for residencies in peripheral hospitals. These students should be identified early during their clinical experience so that attempts to recruit them to the periphery can commence before their specialty and location preferences have fully crystallized. Parallel studies should be performed at additional Israeli medical schools.

Keywords: Medical students, Medical education, Residency, Medical specialty selection, Career choice

\section{Background}

The greatest challenges facing healthcare systems include providing sufficient numbers of primary care and rural physicians [1]. Especially challenging is enticing young physicians to establish primary care practices in rural areas [1, 2]. Among the counties where such challenges exist are the United States, Australia and Canada [1, 3, 4]. Israel suffers from a similar problem with shortages of family medicine specialists in peripheral areas of the country. The latter include the northern and southern regions of the country which encompass the majority of the county's rural districts. These areas chronically suffer from physician shortages, greater infant mortality and lower life expectancy than the rest of the country [5]. In an attempt to remedy this maldistribution, the physicians' union contract of 2011 included both pay increases for practicing in the periphery and onetime monetary incentives for moving and committing to work in the periphery $[6,7]$. This programs also provided incentives for medical students interested in residencies in specialties suffering workforce shortages. The original program excluded family medicine residents from both the incentives for work in the periphery and the incentives to work in distressed specialties. However, in 2015 the Israel Ministry of Health began to provide financial incentives to family medicine residents willing to train in peripheral areas.

The Israeli healthcare system is dependent on primary care physicians to provide comprehensive out-patient care and to serve along with community specialists and internal Health Maintenance Organization regulations and pre-authorization systems as gatekeepers for secondary and tertiary care. These primary care physicians, family medicine specialists, pediatricians, internists and general practitioners, are based in health maintenance organization clinics. Despite family medicine and pediatrics being popular specialties among Israeli medical students, there is still a nationwide shortage of family medicine specialists and pediatricians, especially in the county's peripheral areas. This shortage is predicted to increase as the population grows, ages, and life spans lengthen. Furthermore, the primary care physician population is aging as many physicians who emigrated from the former Soviet Union in the 1990's are reaching retirement age [8]. Therefore, it is necessary to attract more medical students to primary care residencies. To increase recruitment it is important that the medical education and healthcare system leadership better understand the characteristics of students interested in pursuing primary care and how they differ from those without such interests. The leadership needs similar information on students interested in residency positions in the county's periphery.

The present study compared the characteristics of Israeli 5th-year (out of 6 years) medical students interested in primary care residencies and residencies in peripheral areas with those without such interests. The dataset used was collected over a 6-year period from a single Israeli medical school and includes over 500 students. The two hypotheses tested were that the student interest in primary care would be greater among women medical students and that interest in residencies in peripheral areas would be greater among students who attended high school in the northern and southern regions. We also explored whether the one-time monetary grants approved in the 2011 physicians' union contract would encourage students to consider a residency in a peripheral hospital. The ultimate goal was to provide the medical education and healthcare leaderships with the attributes that typify students interested in primary care and/or rural practice. Fifth-year students were studied since our previous study revealed that most had already begun the process of deciding on a specialty [9]. In order for the healthcare leadership to influence specialty decisions, it is important to be cognizant of the thought patterns of the students early in their decision process. 


\section{Methods}

This study included students from 6 consecutive 5th-year classes of the Hebrew University - Hadassah School of Medicine in Jerusalem (2010-2016). It utilized a questionnaire to examine various aspects of the medical specialty selection process. The questionnaire was based on the results of factor analysis from a questionnaire used previously [9]. This permitted us to reduce repeat Likert scale questions thus providing space for new ones that investigated additional issues. Among the new topics examined were the influence of family and colleagues on specialty and residency program decisions as well as the interests of the students in academic pursuits. The questionnaire included multiple choice questions, free-text queries and 5-point Likert scales. In addition to demographic information, the questionnaire elicited information about: (1) Whether the student had already considered a specialty for their residency, which specialty or specialties they were considering (free-text), when they had first considered a specialty and whether and when (prior to beginning medical school or when during the first 5 years of medical school) they had changed their mind; (2) The criteria for choosing a career specialty $\{20$ items, 10 new, 5-point Likert scale\}; (3) The criteria for choosing a residency program $\{20$ items, 9 new, 5-point Likert scale $\}$; and (4) The importance of interest in a specific specialty when choosing a residency $\{3$ new items, multiple choice\}. (5) Whether the one-time monetary grants authorized in the 2011 physicians' union contract would attract them to a residency in a peripheral hospital (1 new item, final 4 classes).

After two small (15 students) preliminary studies designed to identify problems and test the questionnaire's user-friendliness, the questionnaires were distributed to the 5th year classes of the Hebrew University - Hadassah School of Medicine in Jerusalem during the 2010-2011, 2011-2012, 20122013, 2013-2014, 2014-2015 and 2015-2016 schoolyears. A parallel article examining medical student subgroups also utilized this dataset [10].

\section{Data analysis}

Data were entered into Microsoft Excel (Redmond, WA) spreadsheets and analyses were performed with Systat 12 (San Jose CA).

\section{Primary care}

The dataset was divided into three groups based on the answer to the 5-point Likert scale question: "Are you interested in a primary care residency?" Group 1 included the two points representing positive tendencies; Group 2 included the neutral point; and Group 3 the two points representing negative tendencies. This permitted us to compare students interested and not interested in a primary care residency, while also examining those who were unsure. The results from each of the 6 school-years were compared to determine whether there were differences between years.

\section{Rural (periphery) workforce}

Initial data analysis showed that a significant number of students interested in primary care were interested in a residency in the county's periphery. Therefore, a post-hoc examination was made of the characteristics of students interested in a residency in the country's periphery. The dataset was divided into two groups based on the answer to the 5-point Likert scale question: "Are you interested in a residency in the country's periphery?" Group A included the two points representing positive tendencies while Group B included the two points representing negative tendencies.

\section{Incentives}

The data set was divided into three groups as per the responses to the multiple- choice query "As the result of the union contract of 2011, residents in peripheral hospitals receive a one-time monetary incentive and higher salaries: (1) These incentives attract me to a residency in the periphery (2) I already plan to do a residency in the periphery (3) The incentives do not attract me to a residency in the periphery". The differences between the characteristics of the three groups were determined.

Based on prior research demonstrating significant gender differences associated with specialty selection' an a priori decision was made to separately analyze and compare the male and female data [11].

Responses to multiple choice questions are presented as frequency distributions. When the Likert Scale results were considered continuous variables, statistical analyses were performed using all 5 points. When presented as categorical variables the Likert Scale results were compressed into three categories, (the two points representing negative tendencies and the two points representing positive tendencies were each combined). The percentages of total responses for each of the three categories (positive tendency, middle point and negative tendency) were then computed. 
For continuous data, differences between the groups were analyzed using analysis of variance with Tukey post-hoc tests. Categorical data were analyzed using $\chi^{2}$ or Fisher exact tests, as appropriate. A $p$ value $<0$. 05 was assumed to represent statistical significance. Univariate linear regression analysis was used to examine the association between the answers to two queries. Backward multivariate and logistic regression analyses were performed with the dependent variable being either interest in a residency in primary care or a peripheral hospital. The independent variables were the demographic parameters and specialty and resident selection criteria.

Criteria for specialty and residency program selection were subjected to factor analysis (principal components analysis) using varimax rotation with set eigenvalues of $\geq 1.0$. The data were also analyzed with hierarchal cluster analysis.

The Institutional Review Board of the Hadassah Medical Organization approved this study. Completion of the questionnaire by the student was considered tacit consent.

\section{Results}

Completed questionnaires were returned by 511 of 740 (69\%) 5th-year medical students.

\section{Primary care}

Ninety-eight (19\%) students were interested in pursuing a primary care residency, 184 (36\%) were unsure and 229 (45\%) were not interested. Demographic information is found in Table 1 . Interest in primary care among 5th-year students was stable over the 6-year study period ranging from $17 \%-21 \%$ annually.

Compared to students not interested in primary care, those interested in primary care were significantly less interested in a specialty with procedures/ surgeries and becoming "members of a medical school faculty", while being more inclined towards a specialty dealing with social problems (Tables 2 and 3). When choosing a residency program, students attracted to primary care were more interested than their colleagues in a residency in the country's periphery. Alternately, $42 \%$ of the 48 students who indicated interest in a residency program in the country's periphery were also interested in a primary care residency (Table 2). When asked about specialties they were considering, students interested in primary care expressed significantly more interest in family medicine and pediatrics than those not interested in primary care. Among the latter, none were considering family medicine (Table 1 ).

As can be seen in Tables 1 and 2 (Appendix A), there was similar interest in a primary care residency between female (17\%) and male (21\%) students. Comparisons between men and women students interested in primary care showed that women rated lifestyle issues, such as family time, more highly than men and were more interested in pediatrics (Tables 1 and 2, Appendix A).

\section{Rural (periphery) workforce}

Differences between students expressing and not interested in a residency in the country's periphery are found in Table 4 (Appendix B). Results of multivariate and logistic regression analyses are in Table 3.

Interactions between the replies to the questions "Are you interested in a residency in the country's periphery?" and "Are you interested in a primary care residency?" revealed that $4 \%$ of all the students were interested in both a primary care residency and a residency in the periphery (Appendix C).

\section{Incentives}

Responses to the query about monetary incentives for a residency in peripheral hospitals are in Tables 5 (Appendix D). Thirty percent of the students reported that the incentives interested them, while another $6 \%$ had already decided to do a residency in the periphery. The relationships between the responses to this question and those to the query "are you interested in a residency in the country's periphery?" revealed that $82 \%$ of the students who replied they were not attracted by the incentives indicated that they were not interested in a residency in the periphery while those that reported that the incentives interested them showed less aversion (20\% negative tendency and $54 \%$ positive tendency) to a residency in the periphery.

\section{Discussion}

The present study identified several medical student characteristics associated with interest in a primary care residency and those interested in a residency in the periphery among 5th year students at the Hadassah-Hebrew University Medical School.

\section{Primary care}

There were many differences between 5th-year Israeli medical students interested and not interested in a 
Table 1 Primary care - demographic and other information

\begin{tabular}{|c|c|c|c|c|c|c|c|c|}
\hline & & \multirow{2}{*}{$\begin{array}{l}\text { Primary } \\
\text { care }\end{array}$} & \multirow[t]{2}{*}{ Undecided } & \multirow{2}{*}{$\begin{array}{l}\text { No primary } \\
\text { care }\end{array}$} & \multirow{2}{*}{$\begin{array}{l}\text { Primary care vs } \\
\text { no primary care }\end{array}$} & \multicolumn{2}{|c|}{ Primary care } & \multirow{2}{*}{$\begin{array}{l}\text { Females } \\
\text { vs males }\end{array}$} \\
\hline & & & & & & Females & Males & \\
\hline \multicolumn{2}{|l|}{ N } & 98 & 184 & 229 & & 41 & 57 & \\
\hline \multicolumn{2}{|l|}{ Female } & $42 \%$ & $51 \%$ & $49 \%$ & & & & \\
\hline \multicolumn{2}{|l|}{ Male } & $58 \%$ & $50 \%$ & $51 \%$ & NS & & & \\
\hline \multirow[t]{6}{*}{ Age (years) } & $18-20$ & $0 \%$ & $0 \%$ & $0.4 \%$ & & $0 \%$ & $0 \%$ & \\
\hline & $21-23$ & $25 \%$ & $14 \%$ & $17 \%$ & & $27 \%$ & $23 \%$ & \\
\hline & $24-26$ & $19 \%$ & $31 \%$ & $29 \%$ & & $34 \%$ & $9 \%$ & \\
\hline & $27-29$ & $37 \%$ & $42 \%$ & $39 \%$ & & $27 \%$ & $44 \%$ & \\
\hline & $30-32$ & $14 \%$ & $10 \%$ & $12 \%$ & & $10 \%$ & $18 \%$ & \\
\hline & +32 & $5 \%$ & $4 \%$ & $3 \%$ & NS & $2 \%$ & $7 \%$ & $p<0.01$ \\
\hline \multirow[t]{3}{*}{ Marital status } & Single & $70 \%$ & $63 \%$ & $73 \%$ & & $66 \%$ & $72 \%$ & \\
\hline & Married & $30 \%$ & $36 \%$ & 26. \% & & $34 \%$ & $26 \%$ & \\
\hline & Divorced & $1 \%$ & $1 \%$ & $0.4 \%$ & NS & $0 \%$ & $2 \%$ & NS \\
\hline \multirow[t]{2}{*}{ Thought of a specialty when started } & Yes & $85 \%$ & $79 \%$ & $82 \%$ & & $85 \%$ & $84 \%$ & \\
\hline & No & $15 \%$ & $21 \%$ & $18 \%$ & NS & $15 \%$ & $16 \%$ & NS \\
\hline \multirow[t]{6}{*}{ When did you start thinking of a specialty? } & Pre - med school & $25 \%$ & $29 \%$ & $26 \%$ & & $27 \%$ & $23 \%$ & \\
\hline & Year 1 & $4 \%$ & $4 \%$ & $4 \%$ & & $6 \%$ & $2 \%$ & \\
\hline & Year 2 & $4 \%$ & $4 \%$ & $2 \%$ & & $3 \%$ & $4 \%$ & \\
\hline & Year 3 & $4 \%$ & $3 \%$ & $5 \%$ & & $0 \%$ & $6 \%$ & \\
\hline & Year 4 & $56 \%$ & $42 \%$ & $48 \%$ & & $62 \%$ & $51 \%$ & \\
\hline & Year 5 & $9 \%$ & $19 \%$ & $16 \%$ & NS & $3 \%$ & $13 \%$ & NS \\
\hline \multirow[t]{2}{*}{ Have you changed your mind? } & Yes & $61 \%$ & $59 \%$ & $63 \%$ & & $59 \%$ & $62 \%$ & \\
\hline & No & $40 \%$ & $41 \%$ & $38 \%$ & NS & $41 \%$ & $38 \%$ & NS \\
\hline \multirow[t]{5}{*}{ When did you change your mind? } & Year 1 & $0 \%$ & $0 \%$ & $2 \%$ & & $0 \%$ & $0 \%$ & \\
\hline & Year 2 & $0 \%$ & $3 \%$ & $1 \%$ & & $0 \%$ & $0 \%$ & \\
\hline & Year 3 & $2 \%$ & $1 \%$ & $2 \%$ & & $0 \%$ & $4 \%$ & \\
\hline & Year 4 & $41 \%$ & $44 \%$ & $42 \%$ & & $35 \%$ & $46 \%$ & \\
\hline & Year 5 & $57 \%$ & $52 \%$ & $54 \%$ & NS & $65 \%$ & $50 \%$ & NS \\
\hline \multirow[t]{7}{*}{ Specialties under consideration } & Family medicine & $16 \%$ & $2 \%$ & $0 \%$ & & $14 \%$ & $17 \%$ & \\
\hline & Pediatrics & $41 \%$ & $42 \%$ & $24 \%$ & & $54 \%$ & $30 \%$ & \\
\hline & Internal med & $26 \%$ & $30 \%$ & $32 \%$ & & $26 \%$ & $26 \%$ & \\
\hline & Ob/Gyn & $19 \%$ & $20 \%$ & $22 \%$ & & $34 \%$ & $7 \%$ & \\
\hline & Emergency med & $4 \%$ & $0 \%$ & $2 \%$ & & $0 \%$ & $7 \%$ & \\
\hline & Surgical* & $20 \%$ & $31 \%$ & $31 \%$ & & $9 \%$ & $30 \%$ & \\
\hline & Other & $22 \%$ & $44 \%$ & $39 \%$ & $p<0.01$ & $20 \%$ & $30 \%$ & $p<0.01$ \\
\hline \multirow[t]{2}{*}{ High school location } & Israel & $97 \%$ & $97 \%$ & $96 \%$ & & $95 \%$ & $98 \%$ & \\
\hline & Other & $3 \%$ & $3 \%$ & $4 \%$ & NS & $5 \%$ & $2 \%$ & NS \\
\hline \multirow[t]{5}{*}{ High school location in israel } & Jerusalem & $32 \%$ & $25 \%$ & $23 \%$ & & $26 \%$ & $36 \%$ & \\
\hline & Central & $29 \%$ & $37 \%$ & $44 \%$ & & $31 \%$ & $29 \%$ & \\
\hline & North & $26 \%$ & $28 \%$ & $23 \%$ & & $33 \%$ & $21 \%$ & \\
\hline & South & $13 \%$ & $9 \%$ & $10 \%$ & & $10 \%$ & $14 \%$ & \\
\hline & Other & $0 \%$ & $1 \%$ & $1 \%$ & NS & $0 \%$ & $0 \%$ & NS \\
\hline \multirow[t]{2}{*}{ Future residency location } & Israel & $98 \%$ & $98 \%$ & $99 \%$ & & $97 \%$ & $98 \%$ & \\
\hline & Other & $2 \%$ & $2 \%$ & $1 \%$ & NS & $3 \%$ & $2 \%$ & NS \\
\hline
\end{tabular}


Table 1 Primary care - demographic and other information (Continued)

\begin{tabular}{|c|c|c|c|c|c|c|c|c|}
\hline & & \multirow{2}{*}{$\begin{array}{l}\text { Primary } \\
\text { care }\end{array}$} & \multirow[t]{2}{*}{ Undecided } & \multirow{2}{*}{$\begin{array}{l}\text { No primary } \\
\text { care }\end{array}$} & \multirow{2}{*}{$\begin{array}{l}\text { Primary care vs } \\
\text { no primary care }\end{array}$} & \multicolumn{2}{|c|}{ Primary care } & \multirow{2}{*}{$\begin{array}{l}\text { Females } \\
\text { vs males }\end{array}$} \\
\hline & & & & & & Females & Males & \\
\hline \multirow[t]{5}{*}{ Perferred residency location in Israel } & Jerusalem & $28 \%$ & $18 \%$ & $23 \%$ & & $23 \%$ & $31 \%$ & \\
\hline & Central & $22 \%$ & $32 \%$ & $34 \%$ & & $13 \%$ & $29 \%$ & \\
\hline & North & $17 \%$ & $11 \%$ & $9 \%$ & & $23 \%$ & $12 \%$ & \\
\hline & South & $1 \%$ & $1 \%$ & $2 \%$ & & $3 \%$ & $0 \%$ & \\
\hline & Don't know & $33 \%$ & $39 \%$ & $32 \%$ & $p<0.05$ & $39 \%$ & $29 \%$ & $p<0.01$ \\
\hline
\end{tabular}

*Surgical specialties

primary care residency. Students inclined toward primary care were more interested in lifestyle: spending time with their families, working limited hours and working only during the daytime. This importance of lifestyle was more pronounced in female than male students. Students inclined toward primary care were less interested in academic pursuits, such as being academic faculty members. Reduced interest in academic activities was also observed among Japanese medical students with preferences for

Table 2 Primary care - selection criteria

\begin{tabular}{|c|c|c|c|c|c|c|c|}
\hline & \multirow{2}{*}{$\begin{array}{l}\text { Primary } \\
\text { care }\end{array}$} & \multirow[t]{2}{*}{ Undecided } & \multirow{2}{*}{$\begin{array}{l}\text { No primary } \\
\text { care }\end{array}$} & \multirow{2}{*}{$\begin{array}{l}\text { Primary care vs } \\
\text { no primary }\end{array}$} & \multicolumn{2}{|c|}{ Primary care } & \multirow[t]{2}{*}{ Females vs males } \\
\hline & & & & & Females & $\overline{\text { Males }}$ & \\
\hline $\mathrm{N}$ & 98 & 184 & 229 & & 41 & 57 & 98 \\
\hline \multicolumn{8}{|l|}{ Criteria for choosing a specialty } \\
\hline Time with family (1) & $76 \%$ & $73 \%$ & $65 \%$ & $p<0.03$ & $85 \%$ & $68 \%$ & $p<0.05$ \\
\hline Specialty with team work & $57 \%$ & $54 \%$ & $45 \%$ & $p<0.04$ & $56 \%$ & $58 \%$ & NS \\
\hline Influence of spouse & $43 \%$ & $43 \%$ & $31 \%$ & $p<0.007$ & $49 \%$ & $39 \%$ & $p<0.05$ \\
\hline Specialty that deals with social issues (3) & $42 \%$ & $35 \%$ & $22 \%$ & $p<0.001$ & $51 \%$ & $35 \%$ & $p<0.009$ \\
\hline Daytime work only (1) & $26 \%$ & $29 \%$ & $16 \%$ & $p<0.03$ & $34 \%$ & $19 \%$ & $p<0.03$ \\
\hline Work only in the community & $9 \%$ & $3 \%$ & $4 \%$ & $p<0.001$ & $12 \%$ & $7 \%$ & NS \\
\hline Procedures/surgery & $43 \%$ & $41 \%$ & $57 \%$ & $p<0.01$ & $34 \%$ & $49 \%$ & $p<0.02$ \\
\hline High salary & $36 \%$ & $48 \%$ & $51 \%$ & $p<0.002$ & $34 \%$ & $38 \%$ & NS \\
\hline Opportunity for research (2) & $34 \%$ & $37 \%$ & $44 \%$ & $p<0.05$ & $27 \%$ & $39 \%$ & NS \\
\hline Academic faculty member & $19 \%$ & $26 \%$ & $30 \%$ & $p<0.05$ & $24 \%$ & $16 \%$ & NS \\
\hline \multicolumn{8}{|l|}{ Criteria for choosing a residency } \\
\hline Much supervision by senior physicians & $53 \%$ & $45 \%$ & $37 \%$ & $p<0.001$ & $68 \%$ & $42 \%$ & $p<0.04$ \\
\hline Limited work hours & $29 \%$ & $30 \%$ & $17 \%$ & $p<0.001$ & $33 \%$ & $27 \%$ & NS \\
\hline Short residency $(<4.5$. years) & $28 \%$ & $15 \%$ & $12 \%$ & $p<0.001$ & $29 \%$ & $26 \%$ & NS \\
\hline Much clinic time (2) & $23 \%$ & $16 \%$ & $5 \%$ & $p<0.001$ & $20 \%$ & $25 \%$ & NS \\
\hline Hospital in the periphery (3) & $19 \%$ & $7 \%$ & $6 \%$ & $p<0.001$ & $20 \%$ & $19 \%$ & NS \\
\hline Leading department $(1)^{a}$ & $67 \%$ & $77 \%$ & $79 \%$ & $p<0.03$ & $71 \%$ & $65 \%$ & NS \\
\hline Large hospitial & $52 \%$ & $55 \%$ & $62 \%$ & $p<0.04$ & $59 \%$ & $47 \%$ & NS \\
\hline Family living location & $78 \%$ & $75 \%$ & $64 \%$ & NS & $88 \%$ & $70 \%$ & $p<0.05$ \\
\hline Making clinical decisions on your own & $66 \%$ & $50 \%$ & $55 \%$ & NS & $56 \%$ & $74 \%$ & $p<0.05$ \\
\hline Pre-determined work hours (2) & $47 \%$ & $48 \%$ & $35 \%$ & NS & $59 \%$ & $39 \%$ & $p<0.05$ \\
\hline Influence of family & $42 \%$ & $37 \%$ & $31 \%$ & NS & $54 \%$ & $33 \%$ & $p<0.01$ \\
\hline Many on-call shifts & $10 \%$ & $11 \%$ & $12 \%$ & NS & $5 \%$ & $14 \%$ & $p<0.03$ \\
\hline
\end{tabular}

Percent of "agree" and "agree much" responses on 5-point Likert Scale Numbers in parenthesis are the results of factor analysis ${ }^{a}$ clusters per cluster analysis

Bold result indicates the higher value in a statistically significant pair 
Table 3 Regression analysis

\begin{tabular}{|c|c|c|c|c|c|}
\hline \multirow[t]{2}{*}{ Demographics/criteria } & \multirow{2}{*}{$\begin{array}{l}\text { Multivariable Backward } \\
\text { regression } \\
\text { coefficient }\end{array}$} & \multirow[b]{2}{*}{$p$} & \multicolumn{3}{|c|}{ Backward logistic regression } \\
\hline & & & Odds ratio & $95 \%$ confidence limits & $P$ \\
\hline Interest in a primary care residency & $r=0.43$ & & & & \\
\hline Male gender & 0.264 & 0.015 & 2.251 & $1.195-4.241$ & 0.012 \\
\hline \multicolumn{6}{|l|}{ Criteria for choosing a specialty } \\
\hline Opportunity for research & -0.096 & 0.033 & 0.755 & $0.578-0.987$ & 0.04 \\
\hline High salary & -0.207 & 0.002 & 0.580 & $0.376-0.893$ & 0.013 \\
\hline Influency of spouse & 0.122 & 0.018 & & & \\
\hline Specialty that deals with social issues & 0.146 & 0.004 & 1.442 & $1.079-1.927$ & 0.013 \\
\hline Work only in the community & 0.278 & 0.001 & & & \\
\hline Wide range of medical problems & & & 1.512 & $1.004-2.277$ & 0.048 \\
\hline Criteria for choosing a residency program & $r=0.45$ & & & & \\
\hline Leading department & -0.125 & 0.053 & 0.581 & $0.395-0.854$ & 0.006 \\
\hline Influence of family & 0.094 & 0.026 & 1.414 & $1.083-1.847$ & 0.011 \\
\hline Short residency ( $<4.5$ years) & 0.159 & 0.001 & 1.524 & $1.122-2.069$ & 0.007 \\
\hline Peripheral hospital & 0.188 & 0.001 & & & \\
\hline Much supervision by senior physicians & 0.202 & 0.001 & 1.836 & $1.340-2.515$ & 0.001 \\
\hline Much clinic time & 0.207 & 0.001 & 1.495 & $1.110-2.013$ & 0.008 \\
\hline Interest in a residency in a peripheral hospital & $r=0.43$ & & & & \\
\hline Older age & 0.157 & 0.004 & 1.732 & $1.144-2.621$ & 0.009 \\
\hline Have considered a specialty & -0.234 & 0.048 & & & \\
\hline High school location (periphery) & 0.138 & 0.002 & 1.546 & $1.089-2.193$ & 0.015 \\
\hline \multicolumn{6}{|l|}{ Criteria for choosing a specialty } \\
\hline Wide range of medical problems & 0.144 & 0.008 & & & \\
\hline Time with family & 0.145 & 0.009 & 1.686 & $1.001-2.839$ & 0.049 \\
\hline Work only in the community & 0.178 & 0.002 & 1.701 & $1.147-2.524$ & 0.008 \\
\hline Influence of spouse & & & 1.488 & $1.019-2.173$ & 0.039 \\
\hline Private practice & -0.109 & 0.012 & & & \\
\hline Specialty that deals with social issues & 0.123 & 0.003 & & & \\
\hline Narrow range of medical problems & 0.298 & 0.001 & 2.156 & $1.377-3.376$ & 0.001 \\
\hline Criteria for choosing a residency program & $r=0.52$ & & & & \\
\hline Specific location in Israel & -0.106 & 0.031 & & & \\
\hline Leading department & -0.280 & 0.001 & 0.480 & $0.091-0.791$ & 0.004 \\
\hline Limited work hours & 0.108 & 0.031 & & & \\
\hline Family living location & 0.115 & 0.046 & & & \\
\hline Many on-call shifts & 0.121 & 0.011 & & & \\
\hline Teaching students & 0.144 & 0.001 & 1.563 & $1.061-2.304$ & 0.024 \\
\hline Physical challenge & 0.157 & 0.001 & & & \\
\hline Primary care & 0.168 & 0.002 & & & \\
\hline Much clinic time & 0.198 & 0.003 & 1.893 & $1.288-2.783$ & 0.001 \\
\hline Much supervision by senior physicians & & & 0.045 & $1.010-2.455$ & 0.045 \\
\hline Influence of family & & & 1.430 & $1.030-1.986$ & 0.022 \\
\hline
\end{tabular}


Table 3 Regression analysis (Continued)

\begin{tabular}{|c|c|c|c|c|c|}
\hline \multirow[t]{2}{*}{ Demographics/criteria } & \multirow{2}{*}{$\begin{array}{l}\text { Multivariable Backward } \\
\text { regression } \\
\text { coefficient }\end{array}$} & \multirow[b]{2}{*}{$p$} & \multicolumn{3}{|c|}{ Backward logistic regression } \\
\hline & & & Odds ratio & 95\% confidence limits & P \\
\hline \multicolumn{6}{|c|}{ Monetary incentives for residency in the periphery } \\
\hline & $r-0.58$ & & & & \\
\hline Opportunity for research & -0.126 & 0.047 & 0.580 & $0.360-0.933$ & 0.025 \\
\hline Specialty advancing rapidly & -0.143 & 0.022 & 0.620 & $0.393-0.90$ & 0.041 \\
\hline Specific location & -0.204 & 0.001 & 0.428 & $0.275-0.667$ & 0.001 \\
\hline Primary care & 0.115 & 0.023 & & & \\
\hline Specialty that deals with social issues & 0.126 & 0.008 & 1.530 & $1.044-2.242$ & 0.029 \\
\hline Peripheral hospital & 0.258 & 0.001 & 2.835 & $1.782-4.511$ & 0.001 \\
\hline
\end{tabular}

family medicine [12]. Lack of interest in academic endeavors is problematic since it reduces the number of family medicine faculty members able to serve as medical student mentors. This lack of mentors might decrease the ability to attract students to the specialty.

It is important to note that the query on the questionnaire was about the broader area of primary care and not specifically about family medicine. Unlike a previous study where we found a femalepredominance among 6th year Israeli medical students interested in family medicine, in the present study we did not find such predominance [13]. Furthermore, the proportion of women medical students interested and not interested in primary care was comparable. Similarly, upon multiple regression analysis, interest in primary care was not associated with being female. We thus failed to prove our hypothesis that interest in primary care would be greater among women than men medical students. This variance with our previous studies is attributable to primary care incorporating general internal medicine, general pediatrics and some aspects of obstetrics/gynecology, in addition to family medicine. We previously found that In Israel, internal medicine and obstetrics/gynecology residencies attract many male students [13]. When asked which specialties they were considering, students interested in primary care listed pediatrics and internal medicine more frequently than family medicine.

In many countries, attracting medical students to primary care careers is a daunting task [14]. The reasons for this difficulty differ between countries [15]. In the United States, the proportion of medical students selecting primary care specialties dropped from $73 \%$ in 1996 to $44 \%$ in 2008, although subsequently there has been some stabilization [16]. Moreover, more internal medicine and pediatric residents are choosing to subspecialize, reducing the numbers entering general internal medicine and pediatric practices [17]. The major reasons cited for the dearth of students entering primary care in the United States are relatively low incomes in the face of high student debt burdens, many administrative tasks and time pressures [16]. Many medical schools have instituted programs to attract more students to primary care, with a multi-year exposure to primary care being more successful than adding a single primary care course to a conventional curriculum [18]. Other countries face similar problems. In Vietnam less than a third of commune (collective farming communities) health stations are staffed by a physician even though the number of medical school graduates almost tripled between 2004 and 2011 [19]. The reasons include poor working conditions, low income and lack of opportunities for career development [19]. In a survey of 9499 South Korean medical students only $2.2 \%$ expressed interest in family medicine [20].

Shortages of primary care physicians are generally attributed to low salaries, lack of prestige and glamor; long hours with frequent on-call responsibilities; and lack of a controllable lifestyle [21, 22]. The situation in Israel differs from other countries in that primary care physicians mainly work in health maintenance clinics, receive salaries comparable to other physicians, have few on-call obligations and have set hours [8]. This was reflected in our previous study where Israeli 6th-year students rated family medicine and pediatrics as specialties with controllable lifestyles and positive relationships between controllable lifestyle and remuneration [13]. This was also found in the present study, where compared to 5th-year students not interested in primary care, those interested in primary care wanted a specialty with time for family involving only daytime work and practice in the community (i.e. outside the hospital). This interest profile was similarly demonstrated by their 
Table 4 Residency in a peripheral hospital

\begin{tabular}{|c|c|c|c|c|}
\hline & & Periphery & $\begin{array}{l}\text { No } \\
\text { periphery }\end{array}$ & $\begin{array}{l}\text { Periphery } \\
\text { vs no } \\
\text { Periphery }\end{array}$ \\
\hline$N$ & & 48 & 382 & \\
\hline \multirow[t]{2}{*}{ Gender } & Female & $45 \%$ & $48 \%$ & \\
\hline & Male & $55 \%$ & $52 \%$ & NS \\
\hline \multirow[t]{6}{*}{ Age (years) } & $18-20$ & $0 \%$ & $0.3 \%$ & \\
\hline & $21-23$ & $17 \%$ & $18 \%$ & \\
\hline & $24-26$ & $19 \%$ & $30 \%$ & \\
\hline & $27-29$ & $42 \%$ & $39 \%$ & \\
\hline & $30-32$ & $17 \%$ & $10 \%$ & \\
\hline & +32 & $6 \%$ & $3 \%$ & NS \\
\hline \multirow[t]{3}{*}{ Marital status } & Single & $63 \%$ & $71 \%$ & \\
\hline & Married & $38 \%$ & $29 \%$ & \\
\hline & Divorced & $0 \%$ & $1 \%$ & NS \\
\hline \multirow[t]{2}{*}{ High school location } & Israel & $100 \%$ & $97 \%$ & \\
\hline & Other & $0 \%$ & $3 \%$ & NS \\
\hline \multirow{5}{*}{$\begin{array}{l}\text { High school location in } \\
\text { Israel }\end{array}$} & Jerusalem & $17 \%$ & $27 \%$ & \\
\hline & Central & $31 \%$ & $42 \%$ & \\
\hline & North & $38 \%$ & $22 \%$ & \\
\hline & South & $15 \%$ & $8 \%$ & \\
\hline & Other & $0 \%$ & $1 \%$ & $p<0.05$ \\
\hline \multirow[t]{2}{*}{ Residency location } & Israel & $100 \%$ & $98 \%$ & \\
\hline & Other & $0 \%$ & $2 \%$ & NS \\
\hline \multirow{5}{*}{$\begin{array}{l}\text { Future residency location } \\
\text { in Israel }\end{array}$} & Jerusalem & $13 \%$ & $26 \%$ & \\
\hline & Central & $16 \%$ & $36 \%$ & \\
\hline & North & $40 \%$ & $7 \%$ & \\
\hline & South & $13 \%$ & $1 \%$ & \\
\hline & Don't know & $18 \%$ & $31 \%$ & $p<0.01$ \\
\hline \multirow[t]{7}{*}{$\begin{array}{l}\text { Specialties under } \\
\text { consideration }\end{array}$} & $\begin{array}{l}\text { Family } \\
\text { medicne }\end{array}$ & $0 \%$ & $2 \%$ & \\
\hline & Pediatrics & $46 \%$ & $30 \%$ & \\
\hline & $\begin{array}{l}\text { Internal } \\
\text { medicine }\end{array}$ & $24 \%$ & $32 \%$ & \\
\hline & Ob/Gyn & $15 \%$ & $21 \%$ & \\
\hline & $\begin{array}{l}\text { Emergency } \\
\text { medicine }\end{array}$ & $5 \%$ & $1 \%$ & \\
\hline & $\begin{array}{l}\text { Surgical } \\
\text { specialties }\end{array}$ & $32 \%$ & $30 \%$ & \\
\hline & $\begin{array}{l}\text { Other } \\
\text { specialties }\end{array}$ & $32 \%$ & $35 \%$ & $p<0.05$ \\
\hline \multicolumn{5}{|c|}{ Criteria for choosing a specialty } \\
\hline Time with family & $85 \%$ & & $66 \%$ & $p<0.001$ \\
\hline Controllable lifestyle & $75 \%$ & & $65 \%$ & $p<0.04$ \\
\hline Influency of spouse & $56 \%$ & & $37 \%$ & $p<0.01$ \\
\hline Specialty that deals & $46 \%$ & & $27 \%$ & $p<0.004$ \\
\hline
\end{tabular}

Table 4 Residency in a peripheral hospital (Continued)

\begin{tabular}{|c|c|c|c|}
\hline & Periphery & $\begin{array}{l}\text { No } \\
\text { periphery }\end{array}$ & $\begin{array}{l}\text { Periphery } \\
\text { vs no } \\
\text { Periphery }\end{array}$ \\
\hline $\begin{array}{l}\text { Work only in the } \\
\text { community }\end{array}$ & $19 \%$ & $3 \%$ & $p<0.001$ \\
\hline $\begin{array}{l}\text { Narrow range of } \\
\text { medical problems }\end{array}$ & $10 \%$ & $2 \%$ & $p<0.001$ \\
\hline Advancing rapidly & $48 \%$ & $62 \%$ & $p<0.05$ \\
\hline $\begin{array}{l}\text { Opportunity for } \\
\text { research }\end{array}$ & $29 \%$ & $41 \%$ & $p<0.03$ \\
\hline \multicolumn{4}{|c|}{ Criteria for choosing a residency program } \\
\hline Family living location & $81 \%$ & $69 \%$ & $p<0.04$ \\
\hline Teaching students & $57 \%$ & $40 \%$ & $p<0.05$ \\
\hline $\begin{array}{l}\text { Pre-determined work } \\
\text { hours }\end{array}$ & $56 \%$ & $41 \%$ & $p<0.05$ \\
\hline Influence of family & $52 \%$ & $33 \%$ & $p<0.02$ \\
\hline $\begin{array}{l}\text { Much supervision } \\
\text { bysenior physicians }\end{array}$ & $50 \%$ & $42 \%$ & $p<0.03$ \\
\hline Primary care & $42 \%$ & $15 \%$ & $p<0.0004$ \\
\hline Limited work hours & $42 \%$ & $21 \%$ & $p<0.03$ \\
\hline Much clinic time & $33 \%$ & $8 \%$ & $p<0.001$ \\
\hline Leading department & $58 \%$ & $79 \%$ & $p<0.0001$ \\
\hline Large hosptial & $47 \%$ & $60 \%$ & $p<0.003$ \\
\hline
\end{tabular}

greater interest in short (in years) residency programs with limited hours and with much time spent in clinics. This grouping of interests indicates a desire for positive life/work balance, something that Israeli non-hospital primary care practice provides. A recent study of Israeli family medicine residents reported similar findings. Specifically, more than $85 \%$ of residents reported that factors that positively influence their choice included the ability to combine work, family, and free time; direct, meaningful contact with patients; the diversity of patients and medical conditions; and attractive working conditions [23]. This interest profile is similar to those reported from other countries among students interested in primary care and family medicine [12, 24]. However, primary care in isolated Israeli rural village has been reported to lead to unclear boundaries between private life and physician roles leading to problems with life/work balance [25]. This may be among the reasons for the shortage of primary care practitioners in the country's periphery.

Rural (periphery) workforce

Worldwide, rural areas often suffer physician shortages. Therefore, in many countries with large rural 
Table $\mathbf{5}$ Incentives - residency in periphery

\begin{tabular}{|c|c|c|c|c|c|c|c|}
\hline & & $\begin{array}{l}\text { 1. Incentive } \\
\text { interests me }\end{array}$ & $\begin{array}{l}\text { 2. Plan peripheral } \\
\text { residency }\end{array}$ & $\begin{array}{l}\text { 3. Incentives don't } \\
\text { interest me }\end{array}$ & 1 vs 3 & 1 vs 2 & 2 vs 3 \\
\hline \multicolumn{2}{|l|}{$\mathrm{N}$} & 106 & 20 & 223 & & & \\
\hline \multicolumn{2}{|l|}{ Percent } & $30 \%$ & $6 \%$ & $64 \%$ & & & \\
\hline \multirow[t]{2}{*}{ Gender } & Female & $48 \%$ & $47 \%$ & $50 \%$ & & & \\
\hline & Male & $52 \%$ & $53 \%$ & $50 \%$ & NS & NS & NS \\
\hline \multirow[t]{6}{*}{ Age (years) } & $18-20$ & $0 \%$ & $0 \%$ & $0 \%$ & & & \\
\hline & $21-23$ & $22 \%$ & $20 \%$ & $19 \%$ & & & \\
\hline & $24-26$ & $27 \%$ & $15 \%$ & $31 \%$ & & & \\
\hline & $27-29$ & $36 \%$ & $50 \%$ & $35 \%$ & & & \\
\hline & $30-32$ & $10 \%$ & $10 \%$ & $11 \%$ & & & \\
\hline & +32 & $5 \%$ & $5 \%$ & $3 \%$ & NS & NS & $p<0.02$ \\
\hline \multirow[t]{3}{*}{ Marital status } & Single & $68 \%$ & $60 \%$ & $72 \%$ & & & \\
\hline & Married & $30 \%$ & $40 \%$ & $27 \%$ & & & \\
\hline & Divorced & $2 \%$ & $0 \%$ & $1 \%$ & NS & $p<0.05$ & $p<0.02$ \\
\hline \multirow[t]{4}{*}{ High school location in Israel } & Jerusalem & $27 \%$ & $5 \%$ & $25 \%$ & & & \\
\hline & Central & $36 \%$ & $32 \%$ & $40 \%$ & & & \\
\hline & South & $10 \%$ & $26 \%$ & $10 \%$ & & & \\
\hline & Other & $1 \%$ & $0 \%$ & $1 \%$ & NS & $p<0.01$ & NS \\
\hline \multirow[t]{5}{*}{ Future residency location in Israel } & Jerusalem & $14 \%$ & $10 \%$ & $29 \%$ & & & \\
\hline & Central & $20 \%$ & $0 \%$ & $41 \%$ & & & \\
\hline & North & $14 \%$ & $55 \%$ & $8 \%$ & & & \\
\hline & South & $0 \%$ & $20 \%$ & $1 \%$ & & & \\
\hline & Don't know & $53 \%$ & $15 \%$ & $22 \%$ & $p<0.03$ & $p<0.01$ & $p<0.01$ \\
\hline \multirow[t]{6}{*}{ Specialties under consideration } & Pediatrics & $39 \%$ & $31 \%$ & $31 \%$ & & & \\
\hline & Internal medicine & $21 \%$ & $6 \%$ & $35 \%$ & & & \\
\hline & Ob/Gyn & $29 \%$ & $19 \%$ & $15 \%$ & & & \\
\hline & Emergency medicine & $0 \%$ & $13 \%$ & $1 \%$ & & & \\
\hline & Surgical specialties & $32 \%$ & $31 \%$ & $29 \%$ & & & \\
\hline & Other specialties & $31 \%$ & $31 \%$ & $37 \%$ & $p<0.04$ & $p<0.01$ & $p<0.02$ \\
\hline \multicolumn{8}{|l|}{ Criteria for choosing a specialty } \\
\hline \multicolumn{2}{|l|}{ Time with family (1) } & $78 \%$ & $60 \%$ & $63 \%$ & $p<0.004$ & $p<0.04$ & NS \\
\hline \multicolumn{2}{|c|}{ Specialty that deals with social issues (3) } & $35 \%$ & $35 \%$ & $25 \%$ & $p<0.02$ & NS & NS \\
\hline \multicolumn{2}{|l|}{ Advancing rapidly (2) } & $51 \%$ & $45 \%$ & $69 \%$ & $p<0.0007$ & NS & $p<0.04$ \\
\hline \multicolumn{2}{|l|}{ Opportunity for research (2) } & $29 \%$ & $5 \%$ & $47 \%$ & $p<0.0001$ & $p<0.04$ & $p<0.0001$ \\
\hline \multicolumn{2}{|l|}{ Controllable lifestyle (1) } & $74 \%$ & $50 \%$ & $66 \%$ & NS & NS & NS \\
\hline \multicolumn{2}{|l|}{ Independent practice } & $54 \%$ & $40 \%$ & $51 \%$ & NS & $p<0.03$ & $p<0.03$ \\
\hline \multicolumn{2}{|l|}{ High salary } & $51 \%$ & $20 \%$ & $45 \%$ & NS & $p<0.006$ & $p<0.01$ \\
\hline \multicolumn{2}{|l|}{ Procedures/surgery } & $45 \%$ & $32 \%$ & $51 \%$ & NS & NS & $p<0.04$ \\
\hline \multicolumn{2}{|l|}{ Private practice } & $37 \%$ & $10 \%$ & $404 \%$ & NS & $p<0.008$ & $p<0.009$ \\
\hline \multicolumn{8}{|l|}{ Criteria for choosing a residency } \\
\hline \multicolumn{2}{|l|}{ Controllable lifestyle } & $73 \%$ & $45 \%$ & $58 \%$ & $p<0.04$ & $p<0.03$ & NS \\
\hline \multicolumn{2}{|l|}{ Primary care } & $27 \%$ & $32 \%$ & $15 \%$ & $p<0.001$ & NS & $p<0.002$ \\
\hline \multicolumn{2}{|l|}{ Hospital in the periphery (3) } & $8 \%$ & $55 \%$ & $4 \%$ & $p<0.001$ & $p<0.0007$ & $p<0.001$ \\
\hline \multicolumn{2}{|l|}{ Intellectual challenge $(1)^{a}$} & $8 \%$ & $60 \%$ & $87 \%$ & $p<0.004$ & NS & $p<0.007$ \\
\hline
\end{tabular}


Table 5 Incentives - residency in periphery (Continued)

\begin{tabular}{|c|c|c|c|c|c|c|}
\hline & $\begin{array}{l}\text { 1. Incentive } \\
\text { interests me }\end{array}$ & $\begin{array}{l}\text { 2. Plan peripheral } \\
\text { residency }\end{array}$ & $\begin{array}{l}\text { 3. Incentives don't } \\
\text { interest me }\end{array}$ & 1 vs 3 & 1 vs 2 & 2 vs 3 \\
\hline Leading department $(1)^{\mathrm{a}}$ & $69 \%$ & $45 \%$ & $85 \%$ & $p<0.0002$ & $p<0.004$ & $p<0.0009$ \\
\hline Specific location & $51 \%$ & $68 \%$ & $70 \%$ & $p<0.0001$ & NS & NS \\
\hline Large hospitial & $50 \%$ & $26 \%$ & $68 \%$ & $p<0.005$ & $p<0.04$ & $p<0.0006$ \\
\hline Opportunity for research & $17 \%$ & $5 \%$ & $29 \%$ & $p<0.02$ & $p<0.01$ & $p<0.0005$ \\
\hline Much supervision by senior physicians & $44 \%$ & $20 \%$ & $46 \%$ & NS & NS & $p<0.04$ \\
\hline
\end{tabular}

Percent of "agree" and "agree much" responses on 5-point Likert Scale

Numbers in parenthesis are the results of factor analysis

${ }^{a}$ Clusters per cluster analysis

Bold result indicares the higher value in a statistically significant pair

areas, such as the United States, Canada and Australia, emphasis has been placed on encouraging more medical students to become rural primary care physicians [26, 27]. To attract students to rural areas, medical schools have programs that expose students to rural practice and have increased the recruitment of students from rural areas [28]. The current study showed that of the more than 500 5th-year Israeli students studied, $8.9 \%$ were considering residency in the country's periphery. This percentage is greater than that reported in our previous study of 5th year students $(4.6 \%)$ and might be attributable to the recent introduction of monetary incentives (one-time grants and salary increases) for physicians choosing to train and practice in the periphery. Shortages of rural physicians frequently includes a lack of primary care physicians; a situation also present in Israel. Among students interested in primary care, 19\% would choose a residency in the periphery. Alternately, among students considering a residency in the periphery, $42 \%$ were interested in primary care. This attraction to primary care among students interested in living in rural areas was also observed among Japanese medical students [29]. However, when we examined our overall student sample, only $3.7 \%$ of the 5th-year students reported interest in both primary care and peripheral residencies.

Regression analysis showed that attending high school in Israel's south and north was associated with interest in residency in the same regions. Notably, significantly more students interested in residencies in the periphery reported that the locale of their family was an important criterion for choosing a residency program. Furthermore, among students who responded to the question concerning the effects of one-time monetary incentives to do a residency in the periphery, "I already plan to do a residency in the periphery", 63\% had gone to high school in the northern or southern areas. Therefore, we confirmed the hypothesis that interest in residencies in peripheral areas is greater among students who attended high school in peripheral regions. Similar observations were made in Kenya, United States, Japan and Australia where students of rural origin were more interested in rural practice $[2,11,28,29]$. These results have potential healthcare policy implications. Firstly, they can contribute to decisions about whether to admit more medical school applicants residing in the periphery in an attempt to reduce the shortage of physicians practicing there. Secondly, the results can also contribute to decisions about whether to include rural medicine rotations during the clinical years to provide all students with exposure to such medical practice [30].

The characteristics of students considering a residency in the periphery were similar to those interested in a primary care residency. Although this may be partially attributable to the many students interested in a primary care residency, it also likely reflects that those interested in residencies in the periphery are more interested in life-work balance and are less interested in a residency in a large hospital and in a leading department.

\section{Incentives}

The last four years of the study provided an opportunity to explore the student's thoughts about the monetary incentives for rural residency included in the 2011 union contract [7]. Although only 6\% were already planning to do a residency in the periphery, $30 \%$ indicated that the incentives induced them to consider a residency there. Fifty-three percent of the latter students reported that they did not yet know 
where they wished to do their residency as opposed to $22 \%$ among those not interested in the incentives. Moreover, there were more differences between the two groups. Students interested in the incentives were more interested in primary care residencies, specialties dealing with social issues, specialties providing family time and residencies affording controllable lifestyles. Alternately, they were less interested in research opportunities, a rapidly advancing specialty and a residency in a leading department in a large hospital. For medical educators and healthcare leaders these results point to a group with distinct characteristics who might be encouraged to join residency programs in the periphery. The challenge is to better characterize this student group, identify them early during their specialty/residency program decision process and provide positive information and counseling about residency programs and lifestyle in the periphery. Thirty percent of the residents working in Israel's periphery reported that the incentives had influenced them greatly, even though initially they had intended to work there [23]. Previous studies have shown that Israeli primary care practitioners in the periphery are more satisfied and had a broader scope of practice than urban practitioners [29, 31, 32]. While the aim is to market residency programs in the periphery to the students, the results of this study show that a third of the students reported that the influence of family was an important part of their residency selection process. Therefore, consideration should be given to including spouses in recruitment efforts.

\section{Strengths and limitations}

The strength of this study is that the large number of students studied allowed us to examine subgroups, such as those interested in primary care. A further strength was its multi-year design showing that the proportion of students interested in primary care and peripheral hospitals remained steady throughout the study.

The major limitation is that the study was performed in a single institution that is located in the center of the country and is focused on academic medicine. Hence the findings are not automatically generalizable to the entire Israeli medical student population, and parallel studies should be carried out at additional Israeli medical schools. ${ }^{1}$

Another limitation is that the study only included Israeli medical students when half the medical internship workforce is comprised of Israeli who graduated from foreign medical schools and immigrants and $58 \%$ of the family medicine residents graduated from such schools [23]. Yet, unlike the foreign graduates who only enter the Israeli healthcare system as interns, having had exposure to the various specialties in foreign healthcare systems during medical school, the Israeli medical students are part of the Israeli healthcare system while they are medical students providing the Israeli healthcare leadership the opportunity to directly expose them to Israeli primary and rural practices early in their clinical experience.

A further limitation is that there might be selection criteria that were not included in the questionnaire. However, both the factor and cluster analyses showed few factors and clusters indicating that a wide-variety of topics were queried.

\section{Conclusions}

The characteristics of students showing interest in primary care and practice in peripheral areas, that were delineated by this study, should aid department heads and residency program directors in identifying potential residents. Moreover, the present study revealed that for Israeli medical students the 5th-year is an important juncture in their choice of a medical specialty. Eighty percent had already considered various specialties. Although, about a quarter had begun their considerations before beginning medical school, the majority had begun during their 4th and 5thyears. Furthermore, $60 \%$ of those who had begun the thought process had already changed their minds. Therefore, the 4th and 5th years of medical school appear to be an opportune time to market the various specialties to medical students and might also be the time to begin informing them about residency programs. Since the students' decisions as to specialty and residency program decisions have major influences on the composition and geographic distribution of the future physician workforce, it is for the healthcare leadership to take the initiative and provide the students with direction, counseling and information to help them with their choices.

\section{Endnote}

${ }^{1}$ Our hypothesis is that the findings for the other medical schools will be similar to what we found for Hebrew University-Hadassah. This is because a) Despite its location in the center of the country, the Hebrew University- Hadassah Medical School draws students from across the country; b) Earlier studies of family medicine specialty preferences found similarities between this medical school and the total for all Israeli medical schools [23]. 


\section{Appendix A}

Table 6 Primary care - selection criteria

\begin{tabular}{|c|c|c|c|c|c|c|c|}
\hline & \multirow{2}{*}{$\begin{array}{l}\text { Primary } \\
\text { care }\end{array}$} & \multirow[t]{2}{*}{ Undecided } & \multirow{2}{*}{$\begin{array}{l}\text { No primary } \\
\text { care }\end{array}$} & \multirow{2}{*}{$\begin{array}{l}\text { Primary care vs } \\
\text { no primary }\end{array}$} & \multicolumn{2}{|c|}{ Primary care } & \multirow{2}{*}{$\begin{array}{l}\text { Females } \\
\text { vs males }\end{array}$} \\
\hline & & & & & Females & Males & \\
\hline N & 98 & 184 & 229 & & 41 & & \\
\hline \multicolumn{8}{|l|}{ Criteria for choosing a specialty } \\
\hline Time with family (1) & $75.51 \%$ & $72.68 \%$ & $65.07 \%$ & $p<0.03$ & $85.37 \%$ & $68.42 \%$ & $p<0.05$ \\
\hline Specialty with team work & $57.14 \%$ & $53.80 \%$ & $44.98 \%$ & $p<0.04$ & $56.10 \%$ & $57.89 \%$ & NS \\
\hline Influence of spouse & $42.55 \%$ & $42.62 \%$ & $31.14 \%$ & $p<0.007$ & $48.65 \%$ & $38.60 \%$ & $p<0.05$ \\
\hline Specialty that deals with social issues (3) & $41.84 \%$ & $34.62 \%$ & $21.93 \%$ & $p<0.001$ & $51.22 \%$ & $35.09 \%$ & $p<0.009$ \\
\hline Daytime work only (1) & $25.51 \%$ & $28.73 \%$ & $16.23 \%$ & $p<0.03$ & $34.15 \%$ & $19.30 \%$ & $p<0.03$ \\
\hline Work only in the community & $9.18 \%$ & $2.73 \%$ & $3.51 \%$ & $p<0.001$ & $12.20 \%$ & $7.02 \%$ & NS \\
\hline Procedures/surgery & $42.86 \%$ & $40.98 \%$ & $56.58 \%$ & $p<0.01$ & $34.15 \%$ & $49.12 \%$ & $p<0.02$ \\
\hline High salary & $36.08 \%$ & $48.09 \%$ & $51.09 \%$ & $p<0.002$ & $34.15 \%$ & $37.50 \%$ & NS \\
\hline Opportunity for research (2) & $33.67 \%$ & $37.16 \%$ & $43.67 \%$ & $p<0.05$ & $26.83 \%$ & $38.60 \%$ & NS \\
\hline Academic faculty member & $19.39 \%$ & $25.68 \%$ & $30.26 \%$ & $p<0.05$ & $24.39 \%$ & $15.79 \%$ & NS \\
\hline Bedside specialty & $93.88 \%$ & $92.93 \%$ & $92.07 \%$ & NS & $92.68 \%$ & $94.74 \%$ & NS \\
\hline Wide range of medical problems & $79.38 \%$ & $71.04 \%$ & $68.12 \%$ & NS & $75.61 \%$ & $82.14 \%$ & NS \\
\hline Controllable lifestyle (1) & $69.39 \%$ & $73.91 \%$ & $64.19 \%$ & NS & $78.05 \%$ & $63.16 \%$ & NS \\
\hline Advancing rapidly (2) & $56.12 \%$ & $61.41 \%$ & $58.77 \%$ & NS & $51.22 \%$ & $59.65 \%$ & NS \\
\hline Independent practice & $51.02 \%$ & $60.11 \%$ & $52.40 \%$ & NS & $56.10 \%$ & $47.37 \%$ & NS \\
\hline Private practice & $39.80 \%$ & $36.96 \%$ & $42.48 \%$ & NS & $41.46 \%$ & $38.60 \%$ & NS \\
\hline Influence of family & $12.37 \%$ & $9.78 \%$ & $8.81 \%$ & NS & $15.00 \%$ & $10.53 \%$ & NS \\
\hline Specialty that my coleagues choose ${ }^{a}$ & $2.06 \%$ & $1.64 \%$ & $0.00 \%$ & NS & $0.00 \%$ & $3.57 \%$ & NS \\
\hline Influence of classmates ${ }^{\mathrm{a}}$ & $2.06 \%$ & $1.63 \%$ & $0.44 \%$ & NS & $0.00 \%$ & $3.57 \%$ & NS \\
\hline Narrow range of medical problems ${ }^{a}$ & $2.04 \%$ & $3.26 \%$ & $3.49 \%$ & NS & $0.00 \%$ & $3.51 \%$ & NS \\
\hline \multicolumn{8}{|l|}{ Criteria for choosing a residency } \\
\hline Much supervision by senior physicians & $53.06 \%$ & $45.11 \%$ & $36.68 \%$ & $p<0.001$ & $68.29 \%$ & $42.11 \%$ & $p<0.04$ \\
\hline Limited work hours & $29.17 \%$ & $29.89 \%$ & $16.67 \%$ & $p<0.001$ & $32.50 \%$ & $26.79 \%$ & NS \\
\hline Short residency (<4.5. Years) & $27.55 \%$ & $15.30 \%$ & $11.79 \%$ & $p<0.001$ & $29.27 \%$ & $26.32 \%$ & NS \\
\hline Much clinic time (2) & $22.45 \%$ & $16.30 \%$ & $4.82 \%$ & $p<0.001$ & $19.51 \%$ & $24.56 \%$ & NS \\
\hline Hospital in the periphery (3) & $19.39 \%$ & $6.52 \%$ & $6.11 \%$ & $p<0.001$ & $19.51 \%$ & $19.30 \%$ & NS \\
\hline Leading department $(1)^{\mathrm{a}}$ & $67.35 \%$ & $77.17 \%$ & $79.04 \%$ & $p<0.03$ & $70.73 \%$ & $64.91 \%$ & NS \\
\hline Large hospitial & $52.04 \%$ & $54.64 \%$ & $61.84 \%$ & $p<0.04$ & $58.54 \%$ & $47.37 \%$ & NS \\
\hline Intellectual challenge $(1)^{a}$ & $83.67 \%$ & $82.07 \%$ & $83.41 \%$ & NS & $87.80 \%$ & $80.70 \%$ & NS \\
\hline Family living location & $77.55 \%$ & $74.86 \%$ & $63.76 \%$ & NS & $87.80 \%$ & $70.18 \%$ & $p<0.05$ \\
\hline Controllable lifestyle & $69.39 \%$ & $70.49 \%$ & $60.70 \%$ & NS & $73.17 \%$ & $66.67 \%$ & NS \\
\hline Making clinical decisions on your own & $66.33 \%$ & $49.46 \%$ & $55.02 \%$ & NS & $56.10 \%$ & $73.68 \%$ & $p<0.05$ \\
\hline Specific location in Israel & $65.31 \%$ & $64.48 \%$ & $63.88 \%$ & NS & $68.29 \%$ & $63.16 \%$ & NS \\
\hline Much "action" & $52.04 \%$ & $33.15 \%$ & $46.49 \%$ & NS & $51.22 \%$ & $52.63 \%$ & NS \\
\hline Pre-determined work hours (2) & $46.94 \%$ & $48.37 \%$ & $35.37 \%$ & NS & $58.54 \%$ & $38.60 \%$ & $p<0.05$ \\
\hline Physical challenge & $47.96 \%$ & $36.96 \%$ & $46.93 \%$ & NS & $41.46 \%$ & $52.63 \%$ & NS \\
\hline Teaching students & $43.88 \%$ & $43.48 \%$ & $43.42 \%$ & NS & $39.02 \%$ & $47.37 \%$ & NS \\
\hline Influence of family & $41.84 \%$ & $37.16 \%$ & $31.00 \%$ & NS & $53.66 \%$ & $33.33 \%$ & $p<0.01$ \\
\hline Opportunity for research & $20.41 \%$ & $25.00 \%$ & $27.95 \%$ & NS & $19.51 \%$ & $21.05 \%$ & NS \\
\hline Many on-call shifts & $10.20 \%$ & $11.41 \%$ & $11.79 \%$ & NS & $4.88 \%$ & $14.04 \%$ & $p<0.03$ \\
\hline
\end{tabular}

Percent of "agree" and "agree much" responses on 5-point Likert Scale

Numbers in parenthesis are the results of factor analysis

${ }^{a}$ Clusters per cluster analysis

Bold results representthe higher value of a statistically significant pair 


\section{Appendix B}

Table $\mathbf{7}$ Residency in a peripheral hospital

\begin{tabular}{|c|c|c|c|}
\hline & Periphery & No periphery & Periphery vs no periphery \\
\hline $\bar{N}$ & 48 & 382 & \\
\hline \multicolumn{4}{|l|}{ Criteria for choosing a specialty } \\
\hline Time with family & $85.42 \%$ & $66.23 \%$ & $p<0.001$ \\
\hline Controllable lifestyle & $75.00 \%$ & $65.01 \%$ & $p<0.04$ \\
\hline Influency of spouse & $56.25 \%$ & $37.40 \%$ & $p<0.01$ \\
\hline Specialty that deals with social issues & $45.83 \%$ & $27.03 \%$ & $p<0.004$ \\
\hline Work only in the community & $18.75 \%$ & $2.62 \%$ & $p<0.001$ \\
\hline Narrow range of medical problems & $10.42 \%$ & $1.83 \%$ & $p<0.001$ \\
\hline Advancing rapidly & $47.92 \%$ & $62.30 \%$ & $p<0.05$ \\
\hline Opportunity for research & $29.17 \%$ & $40.94 \%$ & $p<0.03$ \\
\hline Bedside specialty & $91.67 \%$ & $93.10 \%$ & NS \\
\hline Wide rangeof medical problems & $62.50 \%$ & $72.44 \%$ & NS \\
\hline Independent practice & $60.42 \%$ & $55.24 \%$ & NS \\
\hline Specialty with teamwork & $54.17 \%$ & $47.52 \%$ & NS \\
\hline Procedures/surgery & $50.00 \%$ & $47.24 \%$ & NS \\
\hline High salary & $50.00 \%$ & $47.64 \%$ & NS \\
\hline Private practice & $47.92 \%$ & $41.05 \%$ & NS \\
\hline Daytime work only & $27.08 \%$ & $21.78 \%$ & NS \\
\hline Influence of family & $14.89 \%$ & $10.76 \%$ & NS \\
\hline Academic faculty member & $18.75 \%$ & $28.61 \%$ & NS \\
\hline Influence of classmate & $4.17 \%$ & $1.31 \%$ & NS \\
\hline Specialty that my coleagues choose & $2.08 \%$ & $0.79 \%$ & NS \\
\hline \multicolumn{4}{|l|}{ Criteria for choosing a residency } \\
\hline Family living location & $81.25 \%$ & $68.59 \%$ & $p<0.04$ \\
\hline Teaching students & $56.25 \%$ & $40.05 \%$ & $p<0.05$ \\
\hline Pre-determined work hours & $56.25 \%$ & $40.73 \%$ & $p<0.05$ \\
\hline Influence of family & $52.08 \%$ & $32.98 \%$ & $p<0.02$ \\
\hline Much supervision by senior physicians & $50.00 \%$ & $42.15 \%$ & $p<0.03$ \\
\hline Primary care & $42.22 \%$ & $14.72 \%$ & $p<0.0004$ \\
\hline Limited work hours & $41.67 \%$ & $21.26 \%$ & $p<0.03$ \\
\hline Much clinic time & $33.33 \%$ & $8.38 \%$ & $p<0.001$ \\
\hline Leading department & $58.33 \%$ & $78.59 \%$ & $p<0.0001$ \\
\hline Large hosptial & $46.81 \%$ & $60.05 \%$ & $p<0.003$ \\
\hline Intellectual challenge & $77.08 \%$ & $82.77 \%$ & NS \\
\hline Controllable lifestyle & $77.08 \%$ & $61.56 \%$ & NS \\
\hline Specific location & $75.00 \%$ & $65.79 \%$ & NS \\
\hline Making clinical decisions on your own & $62.50 \%$ & $53.26 \%$ & NS \\
\hline Much "action" & $43.75 \%$ & $40.31 \%$ & NS \\
\hline Physical challenge & $41.67 \%$ & $41.10 \%$ & NS \\
\hline Short residency & $22.92 \%$ & $15.18 \%$ & NS \\
\hline Opportunity for research & $20.83 \%$ & $25.85 \%$ & NS \\
\hline Many on-call shifts & $16.67 \%$ & $9.14 \%$ & NS \\
\hline
\end{tabular}

Percent of "agree" and "agree much" responses on 5-point Likert Scale Numbers in parenthesis are the results of factor analysis

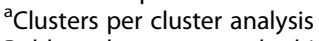

Bold results represent the higher value of a statisitcally significant pair 


\section{Appendix C}

Table $\mathbf{8}$ In "residency in the periphery" and "primary care residency"

\begin{tabular}{|c|c|c|c|c|c|}
\hline \multicolumn{6}{|c|}{ Residency in the periphery } \\
\hline & & Not intersted & Neutral & Interested & Total \\
\hline Primary & Not interested & $37.4 \%$ & $4.7 \%$ & $2.7 \%$ & $44.8 \%$ \\
\hline Care & Neutral & $22.7 \%$ & $11.0 \%$ & $2.3 \%$ & $36.0 \%$ \\
\hline \multirow[t]{2}{*}{ Residency } & Interested & $10.4 \%$ & $5.1 \%$ & $3.7 \%$ & $19.2 \%$ \\
\hline & Total & $70.5 \%$ & $20.7 \%$ & $8.8 \%$ & \\
\hline
\end{tabular}

\section{Appendix D}

Table 9 Primary care - selection criteria

\begin{tabular}{|c|c|c|c|c|c|c|}
\hline & $\begin{array}{l}1 \\
\text { Incentive } \\
\text { interests me } \\
\end{array}$ & $\begin{array}{l}2 \\
\text { Plan peripheral } \\
\text { residency }\end{array}$ & $\begin{array}{l}3 \\
\text { Incentives don't } \\
\text { interest me }\end{array}$ & 1 vs 3 & 1 vs 2 & 2 vs 3 \\
\hline $\mathrm{N}$ & 106 & 20 & 223 & & & \\
\hline \multicolumn{7}{|l|}{ Criteria for choosing a specialty } \\
\hline Time with family (1) & $78.30 \%$ & $60.00 \%$ & $63.39 \%$ & $p<0.004$ & $p<0.04$ & NS \\
\hline Specialty that deals with social issues (3) & $35.24 \%$ & $35.00 \%$ & $25.11 \%$ & $p<0.02$ & NS & NS \\
\hline Advancing rapidly (2) & $51.43 \%$ & $45.00 \%$ & $68.75 \%$ & $p<0.0007$ & NS & $p<0.04$ \\
\hline Opportunity for research (2) & $29.25 \%$ & $5.00 \%$ & $46.64 \%$ & $p<0.0001$ & $p<0.04$ & $p<0.0001$ \\
\hline Bedside specialty & $95.28 \%$ & $95.00 \%$ & $92.79 \%$ & NS & NS & NS \\
\hline Wide range of medical problems & $73.58 \%$ & $63.16 \%$ & $73.66 \%$ & NS & NS & NS \\
\hline Controllable lifestyle (1) & $73.58 \%$ & $50.00 \%$ & $66.07 \%$ & NS & NS & NS \\
\hline Independent practice practice & $53.77 \%$ & $40.00 \%$ & $51.28 \%$ & NS & $p<0.03$ & $p<0.03$ \\
\hline High salary & $50.48 \%$ & $20.00 \%$ & $45.09 \%$ & NS & $p<0.006$ & $p<0.01$ \\
\hline Specialty with team work & $48.11 \%$ & $55.00 \%$ & $50.89 \%$ & NS & NS & NS \\
\hline Procedures/surgery & $45.28 \%$ & $31.58 \%$ & $50.67 \%$ & NS & NS & $p<0.04$ \\
\hline Influence of spouse & $41.35 \%$ & $45.00 \%$ & $34.84 \%$ & NS & NS & NS \\
\hline Private practice & $36.79 \%$ & $10.00 \%$ & $39.64 \%$ & NS & $p<0.008$ & $p<0.009$ \\
\hline Daytime work only (1) & $23.30 \%$ & $21.05 \%$ & $19.20 \%$ & NS & NS & NS \\
\hline Academic faculty member & $22.86 \%$ & $10.53 \%$ & $31.25 \%$ & NS & NS & NS \\
\hline Influence of family & $9.43 \%$ & $10.00 \%$ & $11.21 \%$ & NS & NS & NS \\
\hline Work only in the community & $4.72 \%$ & $10.00 \%$ & $3.14 \%$ & NS & NS & NS \\
\hline Narrow range of medical problems ${ }^{a}$ & $4.72 \%$ & $5.00 \%$ & $2.68 \%$ & NS & NS & NS \\
\hline Specialty that my coleagues choose ${ }^{a}$ & $1.90 \%$ & $5.00 \%$ & $0.90 \%$ & NS & NS & NS \\
\hline Influence of classmates ${ }^{a}$ & $0.95 \%$ & $0.00 \%$ & $1.34 \%$ & NS & NS & NS \\
\hline \multicolumn{7}{|l|}{ Criteria for choosing a residency } \\
\hline Controllable lifestyle & $73.33 \%$ & $45.00 \%$ & $58.12 \%$ & $p<0.04$ & $p<0.03$ & NS \\
\hline Primary care & $27.18 \%$ & $31.58 \%$ & $15.32 \%$ & $p<0.001$ & NS & $p<0.002$ \\
\hline Hospital in the periphery (3) & $7.55 \%$ & $55.00 \%$ & $3.85 \%$ & $p<0.001$ & $p<0.0007$ & $p<0.001$ \\
\hline Intellectual challenge $(1)^{\mathrm{a}}$ & $80.19 \%$ & $60.00 \%$ & $87.05 \%$ & $p<0.004$ & NS & $p<0.007$ \\
\hline Leading department $(1)^{a}$ & $68.87 \%$ & $45.00 \%$ & $84.82 \%$ & $p<0.0002$ & $p<0.004$ & $p<0.0009$ \\
\hline Specific location & $50.94 \%$ & $68.42 \%$ & $69.82 \%$ & $p<0.0001$ & NS & NS \\
\hline Large hospitial & $50.00 \%$ & $26.32 \%$ & $67.71 \%$ & $p<0.005$ & $p<0.04$ & $p<0.0006$ \\
\hline Opportunity for research & $16.98 \%$ & $5.00 \%$ & $29.02 \%$ & $p<0.02$ & $p<0.01$ & $p<0.0005$ \\
\hline Family living location & $68.87 \%$ & $70.00 \%$ & $69.06 \%$ & NS & NS & NS \\
\hline Making clinical decisions on your own & $49.06 \%$ & $50.00 \%$ & $58.04 \%$ & NS & NS & NS \\
\hline
\end{tabular}


Table 9 Primary care - selection criteria (Continued)

\begin{tabular}{|c|c|c|c|c|c|c|}
\hline & $\begin{array}{l}1 \\
\text { Incentive } \\
\text { interests me }\end{array}$ & $\begin{array}{l}2 \\
\text { Plan peripheral } \\
\text { residency }\end{array}$ & $\begin{array}{l}3 \\
\text { Incentives don't } \\
\text { interest me }\end{array}$ & 1 vs 3 & 1 vs 2 & 2 vs 3 \\
\hline Much "action" & $45.28 \%$ & $40.00 \%$ & $43.75 \%$ & NS & NS & NS \\
\hline Much supervision by senior physicians & $43.81 \%$ & $20.00 \%$ & $46.43 \%$ & NS & NS & $p<0.04$ \\
\hline Limited work hours & $43.40 \%$ & $30.00 \%$ & $40.18 \%$ & NS & NS & NS \\
\hline Physical challenge & $42.45 \%$ & $30.00 \%$ & $41.70 \%$ & NS & NS & NS \\
\hline Teaching students & $38.10 \%$ & $50.00 \%$ & $48.21 \%$ & NS & NS & NS \\
\hline Influence of family & $32.38 \%$ & $30.00 \%$ & $34.38 \%$ & NS & NS & $\mathrm{NSb}$ \\
\hline Pre-determined work hours (2) & $25.71 \%$ & $25.00 \%$ & $20.72 \%$ & NS & NS & NS \\
\hline Short residency & $15.09 \%$ & $10.00 \%$ & $16.52 \%$ & NS & NS & NS \\
\hline Much clinic time (2) & $13.33 \%$ & $20.00 \%$ & $9.38 \%$ & NS & NS & NS \\
\hline Many on-call shifts & $12.26 \%$ & $10.00 \%$ & $10.71 \%$ & NS & NS & NS \\
\hline
\end{tabular}

Percent of "agree" and "agree much" responses on 5-point Likert Scale

Numbers in parenthesis are the results of factor analysis

${ }^{a}$ Clusters per cluster analysis

Bold results represent the higher value of a statistically significant pair

\section{Acknowledgements}

The authors thank the many students who participated in this study.

\section{Funding}

Israel National Institute for Health Policy Research.

\section{Availability of data and materials}

The datasets used and/or analyzed during the current study are available from the corresponding author on reasonable request.

\section{Authors' contributions}

CW - Designed study, collected and analyzed data, wrote and edited manuscript. RYZ - Equal contribution with first author - designed study, edited questionnaire, analyzed data, edited, commented and revised manuscript. AA Reviewed and edited questionnaire, reviewed data, edited and commented on manuscript. UA - Reviewed and edited questionnaire, edited and commented on manuscript. HT - Designed study, revised questionnaire, reviewed manuscript. All the authors read and approved the final manuscript.

\section{Ethics approval and consent to participate}

The Institutional Review Board of the Hadassah Medical Organization approved this study. Completion of the questionnaire by the student was considered tacit consent.

\section{Competing interests}

The authors declare that they have no competing interests.

\section{Publisher's Note}

Springer Nature remains neutral with regard to jurisdictional claims in published maps and institutional affiliations.

\section{Author details}

${ }^{1}$ Department of Anesthesiology and Critical Care Medicine, Hadassah-Hebrew University Medical Center, Hebrew University - Hadassah School of Medicine, Kiryat Hadassah, POB 12000, 91120 Jerusalem, Israel. ${ }^{2}$ Hadassah Henrietta Szold School of Nursing, Hebrew University, Jerusalem, Israel. ${ }^{3}$ Department of Obstetrics and Gynecology, Hadassah-Hebrew University Medical Center, Hebrew University Hadassah School of Medicine,
Jerusalem, Israel. ${ }^{4}$ Department of Family Medicine, Ben Gurion University Joyce and Irving Goldman School of Medicine, Be'er Sheva, Israel.

Received: 15 November 2017 Accepted: 25 April 2018

/ Published online: 29 May 2018

\section{References}

1. Allen SM, Ballweg RA, Cosgrove EM, Engle KA, Robinson LR, Rosenblatt RA, Skillman SM, Wenrich MD. Challenges and opportunities in building a sustainable rural primary care workforce in alignment with the Affordable Care Act: the WWAMI program as a case study. Acad Med. 2013;88:1862-9.

2. Duffrin C, Diaz S, Cashion M, Watson R, Cummings D, Jackson N. Factors associated with placement of rural primary care physicians in North Carolina. South Med J. 2014;107:728-33.

3. Sempowski IP. Effectiveness of financial incentives in exchange for rural and underserviced area return-of-service commitments: systematic review of the literature. Can J Rural Med. 2004;9:82-8.

4. Del Mar C. New investments in primary care in Australia. BMC Health Serv Res. 2011;11:39.

5. Israel Ministry of Health: Disparity in health services and ways of coping with it. 2015, Jerusalem; www.health.gov.il/publicationsfiles/inequality-2015. pdf (Accessed 12 Mar 2017).

6. Weil LG, Bin Nun G, Mckee M. Recent physician strike in Israel: a health system under stress? Israel J Health Policy Res. 2013;2:33.

7. Simonstein F. Priorities in the Israeli health care system. Med Health Care Philos. 2013;16:341-7.

8. OECD Reviews of Health Care Quality: Israel 2012: Raising Standards OCED Library http://www.oecd-ilibrary.org/social-issues-migration-health/oecdreviews-of-health-care-quality-israel-2012_9789264029941-en Accessed 26 Feb 2017.

9. Weissman C, Zisk-Rony RY, Schroeder JE, Weiss YG, Avidan A, Elchalal U, Tandeter H. Medical specialty considerations by medical students early in their clinical experience. Isr J Health Policy Res. 2012;1:13.

10. Avidan A, Weissman C, Elchalal U, Tandeter H, Zisk-Rony RY. Medica specialty selection criteria of Israeli medical students early in their clinical experience: subgroups. Isr J Health Policy Res. 2018;7:20.

11. Dossajee H, Obonyo N, Ahmed SM. Career preferences of final year medical students as a medical school in Kenya - a cross sectional study. BMC Medical Education. 2016;16:5. 
12. Kawamoto R, Ninomiya D, Kasai Y, Kusunoki T, Ohtsuka N, Kumagi T, Abe M. Factors associated with the choice of general medicine as a career among Japanese medical students. Med Educ Online. 2016;21:29448.

13. Weissman C, Tandeter H, Zisk-Rony RY, Weiss YG, Elchalal U, Avidan A. Schroeder JE Israeli medical student's perceptions of six key medical specialties. Israel J Health Policy Res. 2013;2:19.

14. Petterson SM, Liaw WR, Tran C, Bazemore AW. Estimating the residency expansion required to avoid projected primary care physician shortages by 2035. Ann Fam Med. 2015;13:107-14.

15. Kringos D, Boerma W, Bourgueil Y, Cartier T, Dedeu T, Hasvold T, Hutchinson A, Lember M, Oleszczyk M, Rotar Pavlic D, Svab I, Tedeschi P, Wilm S, Wilson A, Windak A, Van der Zee J, Groenewegen P. The strength of primary care in Europe: an international comparative study. Br J Gen Pract. 2013;63:e742-50

16. Teng VC, Lin SY. Renewing US medical students' interest in primary care: bridging the role model gap. Postgrad Med. 2014;90:1-2.

17. Messinger CJ, Hafler J, Khan AM, Long T. Recent trends in primary care interest and career choices among medical students at an academic medical institution. Teach Learn Med. 2017;29:42-51.

18. Pfarrwaller E, Sommer J, Chung C, Maisooeuve H, Nendaz M, Perron NJ, Haller DM. Impact of interventions to increase the proportion of medical students choosing a primary career: a systemic review. J Gen Intern Med. 2015;30:1349-58.

19. Giang KB, Minh HV, Hien NV, Ngoc NM, Hinh ND. Knowledge of primary health care and career choice at primary health care settings among final year medical students - challenges to human resources for health in Vietnam. Global Pub Heal. 2017;10(sup 1):S120-30.

20. Kim YY, Kim UN, Kim YS, Lee JS. Factors associated with the specialty choice of Korean medical students: a cross-sectional survey. Hum Resource Health. 2016;14:45.

21. Gold JA, Barg FK, Margo K. Undergraduate students' perspectives on primary care. J Prim Care Comm Health. 2014;5:279-83.

22. Clinite KL, DeZee KJ, Durning SJ, Kogan JR, Blevins T, Chou CL, Diemer G, Dunne DW, Fagan MJ, Hartung PJ, Kazantsev SM, Mechaber HF, Paauw DS, Wong JG, Reddy ST. Lifestyle factors and primary care specialty selection: comparing 2012-2013 graduating and matriculating medical students' thoughts on specialty lifestyle. Acad Med. 2014:89:1483-9.

23. Ashkenazi Y, Gordon M, Yankellevich A, Rosen B. Attracting medical residents to the periphery and to medical specialties in crisis following the 2011 collective agreement. Jerusalem: Myers-JDC-Brookdale Institute Smokler Center for Health Policy Research; 2017.

24. Kiobassa K, Miksch A, Hermann K, Loh A, Szecsenyi J, Joos S, et al. Becoming a general practitioner - which factors have more impact on career choice of medical students? BMC Fam Pract. 2011;12:25.

25. Monnickendam SM, Borkan JM, Matalon A, Zalewski S. Trials and tribulations of country doctors: a qualitative study of doctor-patient relationships in rural Israel. Isr J Med Sci. 1996;32:239-47.

26. Royston PJ, Mathieson K, Leafman J, Ojan-Sheehan O. Medical student characteristics predictive of intent for rural practice. Rural Remote Health. 2012:12:2107.

27. Russell DJ, Wakerman J, Humphreys JS. What is a reasonable length of employment for health workers in Australian rural and remote primary healthcare services? Aust Health Rev. 2013;37:256-61.

28. Puddey IB, Mercer A, Playford DE, Riley GJ. Medical student selection criteria and socio-demographic factors as predictors of ultimately working rurally after graduation. BMC Med Educ. 2017;17:1.

29. Kawamoto R, Uemoto A, Ninomiya D, Hasegawa Y, Ohtsuka N, Kusunoki T, Kumagi T, Abe M. Characteristics of Japanese medical students associated with their intention for rural practice. Rural Remote Health. 2015;15:3112

30. Williamson MI, Wilson R, McKechnie R, Ross J. Does the positive influence of an undergraduate rural placement persist into postgraduate years? Rural Remote Health. 2012;12:2011

31. Fennig S, Yuval D, Greenstein M, Rabin S, Weingarten M. Job satisfaction among certified and non-certified general practitioners. Isr Med Assoc J. 2000:2:823-7.

32. Biderman A, Shvartzman P, Anson O, Almagor G, Grol R. Responsibility taking and role definition in family practice: effect of training and practice setting. Isr J Med Sci. 1996;32:545-50.

\section{Ready to submit your research? Choose BMC and benefit from:}

- fast, convenient online submission

- thorough peer review by experienced researchers in your field

- rapid publication on acceptance

- support for research data, including large and complex data types

- gold Open Access which fosters wider collaboration and increased citations

- maximum visibility for your research: over $100 \mathrm{M}$ website views per year

At BMC, research is always in progress.

Learn more biomedcentral.com/submissions 